\title{
Editorial
}

\section{Recent Advances in Analytical Methods in Mathematical Physics}

\author{
Teoman Özer, ${ }^{1}$ Vladimir B. Taranov, ${ }^{2}$ Roman G. Smirnov, ${ }^{3}$ \\ Thomas Klemas, ${ }^{4}$ Prakash Thamburaja, ${ }^{5}$ Sanith Wijesinghe, ${ }^{6}$ and \\ Burak Polat ${ }^{7}$ \\ ${ }^{1}$ Division of Mechanics, Faculty of Civil Engineering, İstanbul Technical University, Maslak, 34469 \\ İstanbul, Turkey \\ ${ }^{2}$ Department of Plasma Theory, Institute for Nuclear Research, National Academy of Sciences of Ukraine, \\ Kyiv 03680, Ukraine \\ ${ }^{3}$ Department of Mathematics and Statistics, Dalhousie University, Halifax NS, Canada B3H 4R2 \\ ${ }^{4}$ Research Laboratory for Electronics, Massachusetts Institute of Technology, Cambridge, MA 02139, USA \\ ${ }^{5}$ Department of Mechanical Engineering, Faculty of Engineering, National University of Singapore, \\ Singapore 117576 \\ ${ }^{6}$ MITRE Corporation, 3101 Virginia Ave, Silver Spring, MD 20910, USA \\ ${ }^{7}$ Department of Electrical and Electronics Engineering, Faculty of Engineering, Trakya University, 22030 \\ Edirne, Turkey
}

Correspondence should be addressed to Teoman Özer, tozer@itu.edu.tr

Received 22 October 2012; Accepted 22 October 2012

Copyright (C) 2012 Teoman Özer et al. This is an open access article distributed under the Creative Commons Attribution License, which permits unrestricted use, distribution, and reproduction in any medium, provided the original work is properly cited.

\section{Introduction to the Special Issue}

This special issue of the journal Advances in Mathematical Physics was planned to focus on the most recent advances in analytical techniques of particular use to researchers in the field of mathematical physics that covers a very wide area of topics and has a key role in interdisciplinary studies including mathematics, mechanics, and physics. In this special issue, we were particularly interested in receiving novel contributions detailing analytical methods together with appropriate formulations applied to address problems in mathematical physics. We aimed to compile contributions across a variety of disciplines in mathematical physics such as integrability and chaos in dynamical systems, nonlinear partial differential equations, nonlinear problems in mechanics, control theory, geometrical methods, plasma physics, Lie algebras and representation theory, and statistical mechanics.

From different areas of mathematical physics mentioned previously, we have received manuscripts, above thirty, from different countries for consideration in this special issue. After the strict peer-review process the acceptance rate for these manuscripts was $28 \%$. The 
brief descriptions for each accepted manuscript provided by corresponding academic editors are given below.

In their study "Existence and linear stability of equilibrium points in the Robe's restricted three-body problem with oblateness," J. Singh and A. U. Sandah have investigated the positions and linear stability of an infinitesimal body around the equilibrium points in Robe's circular restricted three-body problem when the hydrostatic equilibrium figure of the fluid of the first primary is an oblate spheroid and the second one is an oblate spheroid as well. The equations of motion and the existence of the equilibrium points as well as their linear stability conditions are demonstrated in detail.

In their study "Relativistic double barrier problem with three transmission resonance regions," A. D. Alhaidari et al. have obtained exact scattering solutions of the Dirac equation in $1+1$ dimensions for a double square barrier vector potential. Their main findings are two additional subbarrier transmission resonance regions below the conventional ones. The authors plan to pursue the current line of inquiry to investigate the transport properties of graphene.

In their study "Nonlinear effects of electromagnetic TM wave propagation in anisotropic layer with kerr nonlinearity," Y. G. Smirnov and D. V. Valovik have investigated new eigenvalues and new eigenwaves for the physically important problem of the electromagnetic TM wave propagation through a layer with Kerr nonlinearity. Numerical investigations were performed and the obtained dispersion relation was applied, for example, to the nonlinear metamaterials. The results were compared to the linear theory of the problem under consideration. The approach is restricted to the layer between half spaces with constant permittivity.

In their studies "Combination mode of internal waves generated by surface wave propagating over two muddy sea beds," R.-Y. Yang and H. H. Hwung have investigated the nonlinear response of an initially flat sea bed, with two muddy sections, to a monochromatic surface progressive wave. They showed how resonance of internal waves on a sediment bed can lead to sediment suspension. These results are obtained using a standard perturbation analysis of the weekly nonlinear wavefield system.

In his comprehensive review "Spacetime junctions and the collapse to black holes in higher dimensions," F. C. Mena has focused on recent results of the modeling of gravitational collapse to black holes in higher dimensions with emphasis on cases which involve spacetime junctions with no shell, and in particular nonspherical spacetimes containing a nonzero cosmological constant. The investigation continues with a review of the interesting case of a model of radiating gravitational collapse, in particular, the anisotropic Bizon-ChmajSchmidt (BCS) solution in $4+1$ dimensions, which is compared to data for the SchwarzschildTangherlini solution.

In his study "Mixed initial-boundary value problem for telegraph equation in domain with variable borders," V. A. Ostapenko has provided a novel contribution to exact solutions for a class of mixed initial-boundary value problems for the telegraph equation in an arbitrary domain with time variable borders. While the investigation focuses on the calculation of stress fields in ropes of elevating devices, the developed techniques can be applied to many other physical problems that are represented by similar mixed initial-boundary value problems of hyperbolic type.

In his study "A study on the convergence of series solution of non-Newtonian third grade fluid with variable viscosity: by means of homotopy analysis method," R. Ellahi studied the series solutions of the third-grade non-Newtonian flow. It is mentioned that this flow has a variable viscosity. Since the governing equations of the problem are coupled and highly nonlinear, 
it not possible to find analytical solutions to the problem and hence the homotopy analysis method was applied. Based on this method, the series solutions for the problem are obtained and the convergence of these series solutions and constant and variable viscosity for a thirdgrade flow is presented.

In their studies "Peristaltic transport of a Jeffrey fluid with variable viscosity through a porous medium in an asymmetric channel," A. A. Khan et al. have derived analytic solutions for stream function, velocity, pressure gradient, and pressure rise for peristaltic transport of a Jeffrey fluid with variable viscosity through a porous medium in an asymmetric channel. The variation in flow characteristics are presented graphically as functions of viscosity, Daray number, porosity, amplitude ratio, and Jeffrey fluid parameters for future experimental verification.

In their study "The asymptotic synchronization analysis for two kinds of complex dynamical networks," Z. Tang and J. Feng studied a class of complex networks and obtained sufficient criteria for both time delay-independent and time delay-dependent asymptotic synchronization by using the Lyapunov-Krasovskii stability theorem and linear matrix inequality.

\section{Acknowledgments}

As the guest editors of this special issue, we would like to thank all authors who sent their studies and all referees who spent time in the review process. We wish to thank them again for their contributions and efforts to the success of this special issue.

Teoman Özer

Vladimir B. Taranov

Roman G. Smirnov

Thomas Klemas

Prakash Thamburaja

Sanith Wijesinghe

Burak Polat 


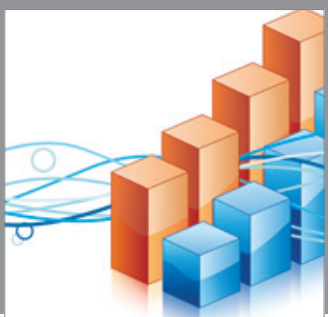

Advances in

Operations Research

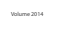

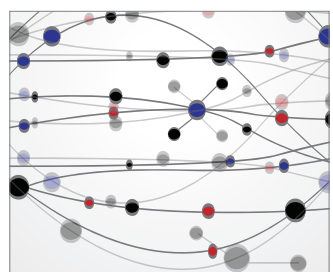

\section{The Scientific} World Journal
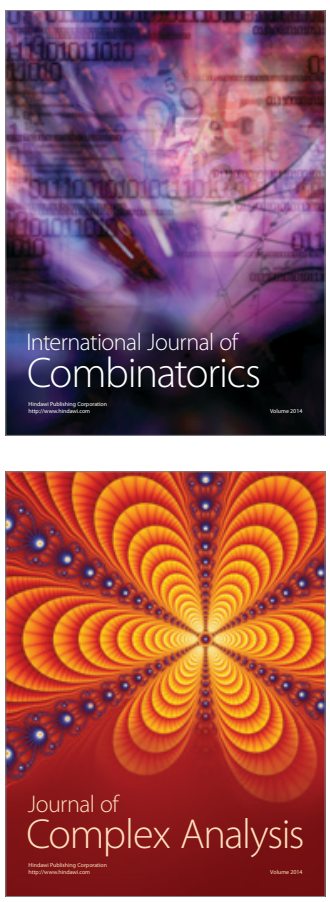

International Journal of

Mathematics and

Mathematical

Sciences
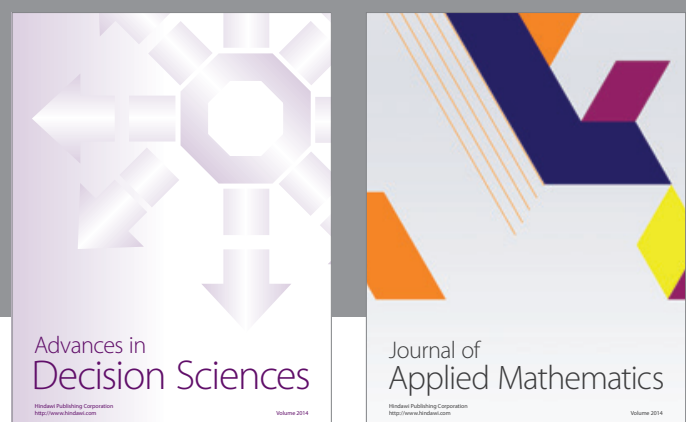

Journal of

Applied Mathematics
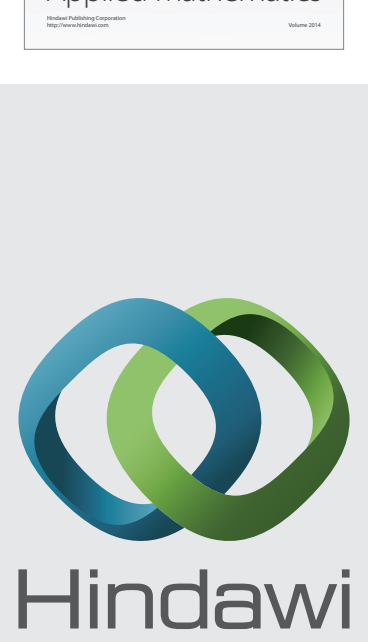

Submit your manuscripts at http://www.hindawi.com
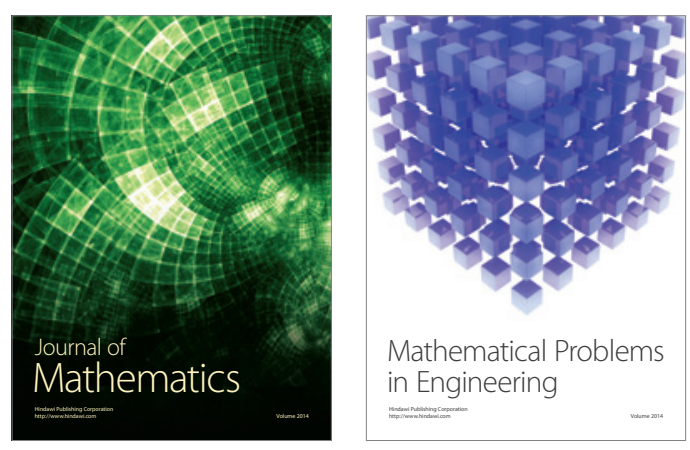

Mathematical Problems in Engineering
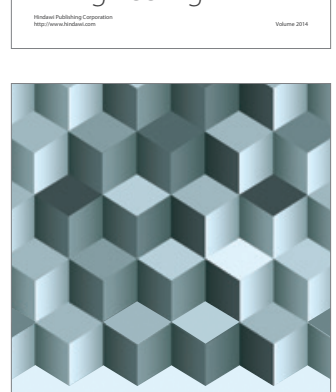

Journal of

Function Spaces
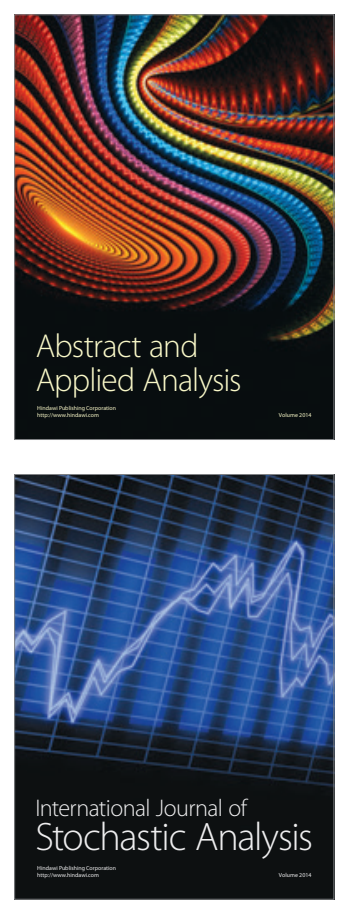

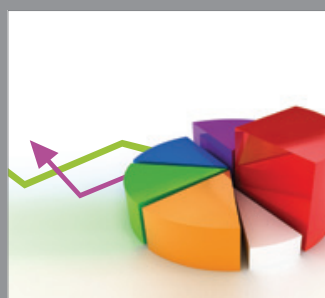

ournal of

Probability and Statistics

Promensencen
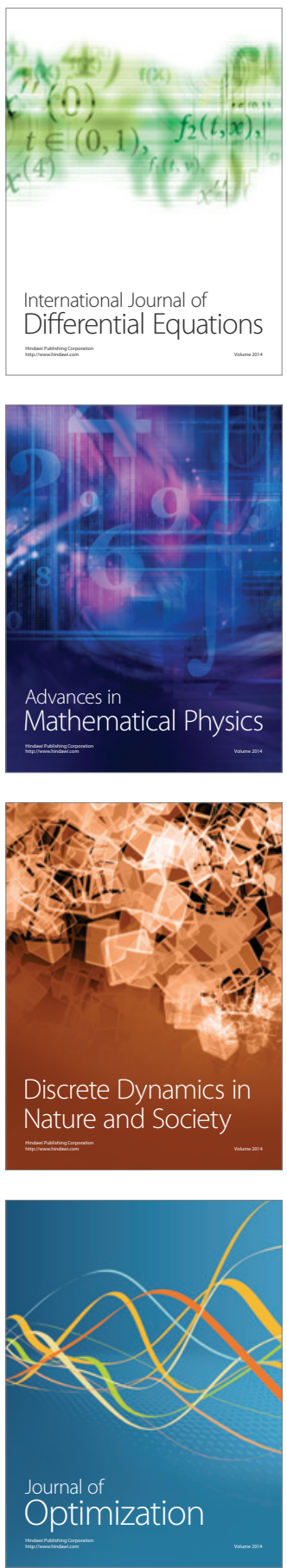